\title{
Bilateral Glaucomatous Optic Neuropathy Caused by Eye Rubbing
}

\author{
Alfonso Savastano $^{a} \quad$ Maria Cristina Savastano $^{b} \quad$ Laura Carlomusto $^{c}$ \\ Silvio Savastanoc \\ ${ }^{a}$ Western Eye Hospital, Imperial College, London, UK; ${ }^{b}$ Università Cattolica del Sacro \\ Cuore, Policlinico Gemelli, Rome, and 'Studio Medico Associato Savastano, Cassino, Italy
}

\section{Key Words}

Advanced glaucoma $\cdot$ Eye rubbing $\cdot$ Optic neuropathy

\begin{abstract}
In this report, we describe a particular condition of a 52-year-old man who showed advanced bilateral glaucomatous-like optic disc damage, even though the intraocular pressure resulted normal during all examinations performed. Visual field test, steady-state pattern electroretinogram, retinal nerve fiber layer and retinal tomographic evaluations were performed to evaluate the optic disc damage. Over a 4-year observational period, his visual acuity decreased to $12 / 20$ in the right eye and counting fingers in the left eye. Visual fields were severely compromised, and intraocular pressure values were not superior to $14 \mathrm{~mm} \mathrm{Hg}$ during routine examinations. An accurate anamnesis and the suspicion of this disease represent a crucial aspect to establish the correct diagnosis. In fact, our patient strongly rubbed his eyes for more than $10 \mathrm{~h}$ per day. Recurrent and continuous eye rubbing can induce progressive optic neuropathy, causing severe visual field damage similar to the pathology of advanced glaucoma.

(c) 2015 S. Karger AG, Basel
\end{abstract}

\section{Introduction}

Eye rubbing can be responsible for several anatomic and functional diseases. Different ocular segments could be implicated, for instance the cornea through keratoconus development or progression [1-5]. Vigorous mechanical eye compression, especially in psychiatric patients (obsessive-compulsive disorder), could induce significant disorders such as vitreous hemorrhage, retinal detachment and even eye rupture (ocular Munchausen's syndrome) [6-8]. Constant high intraocular pressure (IOP) for several hours per day can produce signif-

KARGER 125/s $\quad \begin{aligned} & \text { Alfonso Savastano, MD } \\ & \text { Via A. Savastano n. } 2 \\ & \text { IT-03043 Cassino (Italy) } \\ & \text { E-Mail asavastano21@gmail.com }\end{aligned}$


Savastano et al.: Bilateral Glaucomatous Optic Neuropathy Caused by Eye Rubbing

icant and progressive nerve fiber layer deterioration mimicking glaucomatous disease [9]. The aim of the present study is to describe a case report of bilateral glaucomatous progression in an eye-rubbing patient.

\section{Case Report}

In a 52-year-old man, severe visual acuity loss in the left eye (LE) and progressive sight reduction in the right eye (RE) were observed. The patient showed no history or presence of systemic diseases, and no optic neuritis or any disease involving the macula, retina or visual pathway were observed. Complete ophthalmologic evaluation was performed. The patient showed exotropia due to extreme visual loss in his LE (fig. 1). Best-corrected visual acuity was $12 / 20$ and counting fingers in the RE and LE, respectively, with no refractive error. IOP by daily curve from 8 a.m. to 6 p.m. every $2 \mathrm{~h}$ was measured, resulting in an average value of $12 \pm 2 \mathrm{~mm} \mathrm{Hg}$ (Goldmann applanation tonometry). Central corneal thickness was 554 and $561 \mu \mathrm{m}$ in the RE and LE, respectively (Orbscan II, Bausch and Lomb). Visual field was measured using the Humphrey program 24-2 (Humphrey-Zeiss, Dublin, Calif., USA). A commercially available GLAID (Lace Elettronica, Pisa, Italy, software version 2.1.14) electrophysiology instrument was used to measure the pattern electroretinogram (PERG) response [10]. Steady-state PERG was performed, and amplitude and phase were evaluated. We used the following stimulation settings: round pattern stimuli, $8.14 \mathrm{~Hz}, 1.6^{\circ}$ horizontal bars, $25^{\circ}$ central, skin cup electrodes [11]. Optic nerve head photography was performed (Topcon TRC 50 IX, Topcon Europe Medical, Capelle, The Netherlands) in order to observe the morphological aspect of the optical disc. Retinal tomography (HRTII, Heidelberg Engineering, Heidelberg, Germany) was used after pupil dilation to obtain further objective optic nerve head information. Retinal nerve fiber layer (RNFL) by optical coherence tomography (OCT; Model 3000, Stratus, Carl Zeiss Meditec) was performed under pupil dilatation. According to previous studies, a fast RNFL algorithm was used to obtain RNFL thickness measurements [12, 13]. The patient underwent a complete systemic evaluation by orbit and brain magnetic resonance analysis, electroencephalogram, carotid Doppler and hematological evaluation. All the exams resulted negative for possible systemic disease.

During the previous years, as we could observe from the medical reports that the patient kept, progressive visual acuity and visual field loss was detectable over time. After an accurate anamnesis, the patient's wife mentioned continuous and persistent eye rubbing for more than $10 \mathrm{~h}$ daily for the past 10 years. In particular, she described vigorous compression of both eyes with his hands' thenar eminence (fig. 1). This particular habit caused a temporary blurry vision for several minutes resolving thereafter.

At the observation analysis, both optic discs showed a characteristic aspect with multiple excavation notching that we called 'bubble notching', probably due to a glaucoma-like optic disc damage. The visual field presented an extensive and diffuse defect on $360^{\circ}$ with focal central conservation in the RE, while total visual damage was reported in the LE. The results of steady-state PERG in the RE were: amplitude $0.29 \mu \mathrm{V}$ RMS, phase $0.29 \mathrm{pi}$; and in the LE: amplitude $0.18 \mu \mathrm{V}$ RMS, phase $1.08 \mathrm{pi}$. Analysis of the RNFL by OCT showed a significant average thickness reduction of 66.51 and $67.69 \mu \mathrm{m}$ in the RE and LE, respectively. Retinal tomography evaluation showed the following data in the RE: disc area $=2.518 \mathrm{~mm}^{2}$, cup $/$ disc area ratio $=0.62$; and in the LE: disc area $=1.862 \mathrm{~mm}^{2}$, cup $/$ disc area ratio $=0.696$. The morpho-functional details are reported in fig. 2. 
Savastano et al.: Bilateral Glaucomatous Optic Neuropathy Caused by Eye Rubbing

\section{Conclusions}

Although the damages caused by eye rubbing are reported in several manuscripts, the interest is mostly towards anterior segment involvement. As reported by Slamovits [14], popping eyes can be responsible of progressive bilateral optic neuropathy. Continuous and persistent eye rubbing can cause IOP spikes with values that can reach up to $60-70 \mathrm{~mm} \mathrm{Hg}$, causing a transient arteriolar occlusion (this can be the reason why our patient experienced temporary blurry vision after eye globe compression) [15]. This oxygen deficiency results in optic nerve head stress with secondary nerve fiber damage. Pecora et al. [16] described a case of optic neuropathy caused by eye rubbing after several investigations, some of them extremely invasive such as optic nerve head biopsy. Discriminating eye-rubbing syndromes is not easy, but an accurate anamnesis of the patient and his/her parents could be the only solution to understand an unexplained and progressive loss of sight and visual field associated with glaucoma-like optic damage.

\section{Statement of Ethics}

This case report description followed the Helsinki Declaration criteria, and written informed consent was obtained from the patient for publication of this article and any accompanying images. The Institutional Review Board and Ethics Committee ruled that approval was not required for this study.

\section{Disclosure Statement}

Each author warrants that he/she has no commercial associations that might pose a conflict of interest in connection with the submitted article.

\section{References}

1 Ioannidis AS, Speedwell L, Nischal KK: Unilateral keratoconus in a child with chronic and persistent eye rubbing. Am J Ophthalmol 2005;139:356-357.

2 Yeniad B, Alparslan N, Akarcay K: Eye rubbing as an apparent cause of recurrent keratoconus. Cornea 2009;28:477-479.

-3 McMonnies CW: Mechanisms of rubbing-related corneal trauma in keratoconus. Cornea 2009;28:607-615.

-4 Carlson AN: Expanding our understanding of eye rubbing and keratoconus. Cornea 2010;29:245.

5 Liu WC, Lee SM, Graham AD, Lin MC: Effects of eye rubbing and breath holding on corneal biomechanical properties and intraocular pressure. Cornea 2011;30:855-860.

-6 Rosenberg PN, Krohel GB, Webb RM, Hepler RS: Ocular Munchausen's syndrome. Ophthalmology 1986;93:1120-1123.

-7 Wylegala E, Tarnawska D: Amniotic membrane transplantation with cauterization for keratoconus complicated by persistent hydrops in mentally retarded patients. Ophthalmology 2006;113:561-564. Miller NR: Functional neuro-ophthalmology. Handb Clin Neurol 2011;102:493-513.

-9 Medeiros FA, Alencar LM, Zangwill LM, Sample PA, Weinreb RN: The relationship between intraocular pressure and progressive retinal nerve fiber layer loss in glaucoma. Ophthalmology 2009;116:1125-1133.

$\checkmark 10$ Porciatti V, Ventura LM: Normative data for a user-friendly paradigm for pattern electroretinogram recording. Ophthalmology 2004;111:161-168.

11 Ventura LM, Porciatti V, Ishida K, Feuer WJ, Parrish RK 2nd: Pattern electroretinogram abnormality and glaucoma. Ophthalmology 2005;112:10-19.

12 Zafar S, Gurses-Ozden R, Makornwattana M, et al: Scanning protocol choice affects optical coherence tomography (OCT-3) measurements. J Glaucoma 2004;13:142-144.

13 Hougaard JL, Heijl A, Bengtsson B: Glaucoma detection by Stratus OCT. J Glaucoma 2007;16:302-306. 
Case Reports in

Ophthalmology

\begin{tabular}{l|l}
\hline \multicolumn{2}{l}{ Case Rep Ophthalmol 2015;6:279-283 } \\
\hline DOI: 10.1159/000439163 & $\begin{array}{l}\text { ○ 2015 S. Karger AG, Basel } \\
\text { www.karger.com/cop }\end{array}$ \\
\hline
\end{tabular}

Savastano et al.: Bilateral Glaucomatous Optic Neuropathy Caused by Eye Rubbing

Slamovits T: Popping eyes. Surv Ophthalmol 1989;33:273-280.

Osuagwu UL, Alanazi SA: Eye rubbing-induced changes in intraocular pressure and corneal thickness measured at five locations, in subjects with ocular allergy. Int J Ophthalmol 2015;8:81-88.

16 Pecora L, Sibony P, Fourman S: Eye-rubbing optic neuropathy. Am J Ophthalmol 2002;134:460-461.

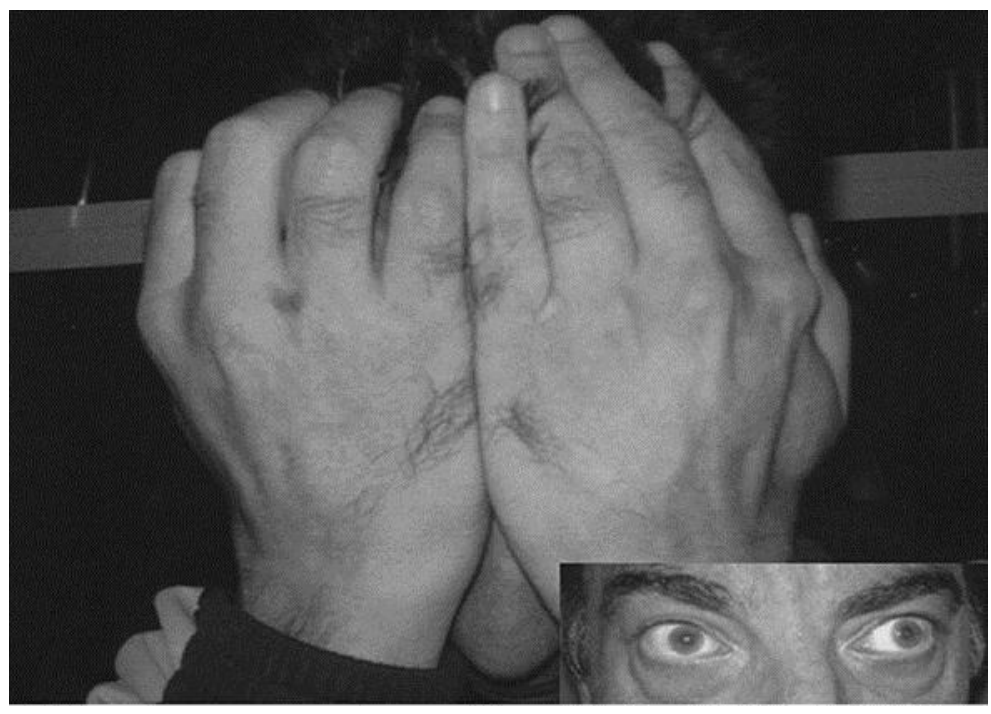

Fig. 1. Photography showing the patient's eye rubbing. The thenar eminence of both hands is vigorously compressed against the eyes. Bottom right: details of exotropia due to severe visual acuity reduction in the left eye. 


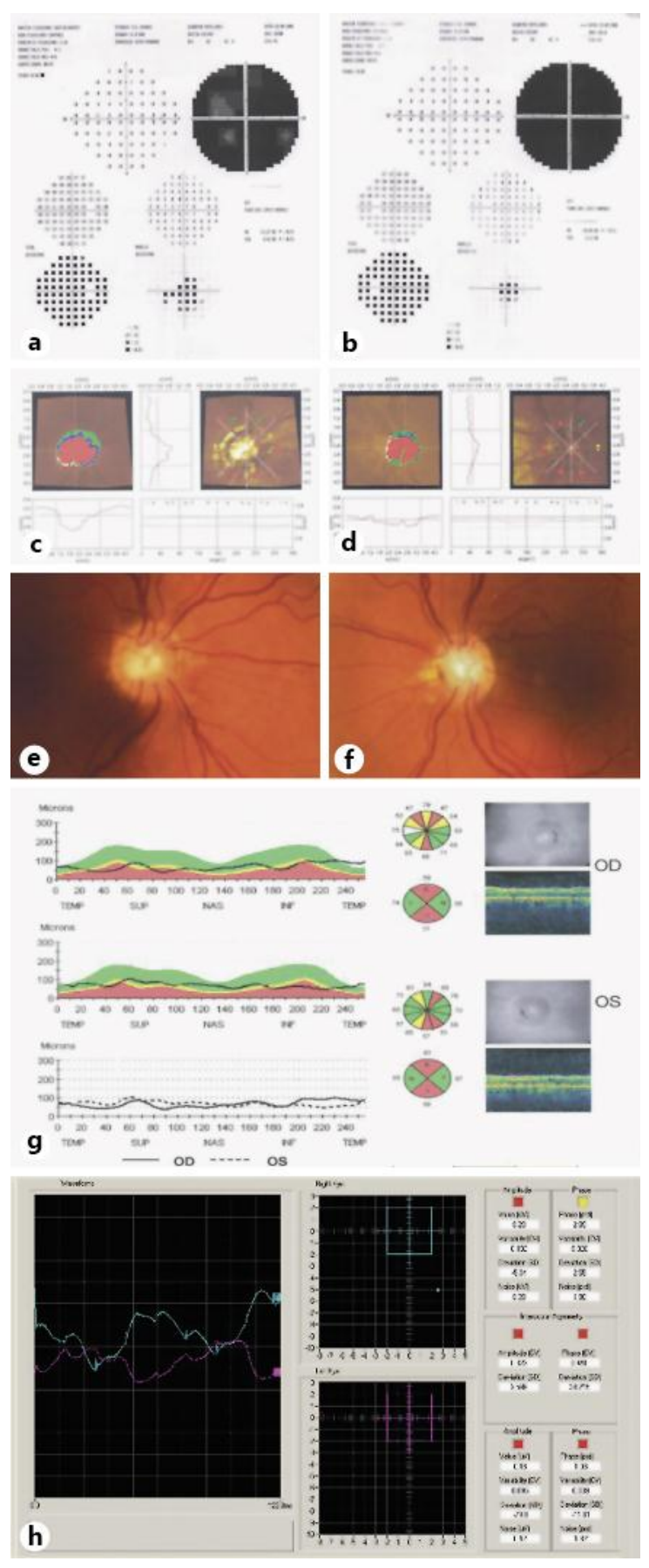

Fig. 2. Visual field showing diffuse defects with focal central conservation in the RE (a) and total visual field deficit in the LE (b). c, d Retinal tomography showing optic nerve head damage in the RE and LE, respectively. e, $\mathbf{f}$ Retinography of both eyes with details of the optic nerve head; a particular aspect with multiple excavation notching can be observed ('bubble notching'). g OCT evaluation revealing the average thickness reduction in both eyes. $\mathbf{h}$ Steady-state PERG analysis showing an amplitude reduction in the RE (blue line) and a very poor signal in the LE (red line). 